\title{
On-Demand QoS Multicast Routing for Triple- Layered LEO/HEO/GEO Satellite IP Networks
}

\author{
Zhizhong Yin \\ The Academy of Equipment Command \& Technology, Beijing, 101416, P.R. China \\ Email: zhizhongyin@126.com \\ Long Zhang and Xianwei Zhou \\ Department of Communication Engineering, School of Computer and Communication Engineering \\ University of Science and Technology Beijing, Beijing, 100083, P.R. China \\ Email: iceberg206@163.com,xwzhouli@sina.com
}

\begin{abstract}
In this paper, for the sake of better global coverage, we introduce a novel triple-layered satellite network architecture including the Geostationary Earth Orbit (GEO), the Highly Elliptical Orbit (HEO), and the Low Earth Orbit (LEO) satellite layers, which provides the near-global coverage with 24 hour uninterrupted over the areas varying from $75^{\circ} \mathrm{S}$ to $90^{\circ} \mathrm{N}$. On the basis of this satellite network architecture, we propose an on-demand QoS multicast routing protocol (ODQMRP) for satellite IP networks using the concept of logical locations to isolate the mobility of LEO and HEO satellites. In ODQMRP, we present two strategies, i.e., the parallel shortest path tree (PSPT) strategy and the least cost tree (LCT) strategy, to create the multicast trees under the condition that the QoS requirements, containing the delay constraint, and the available bandwidth constraint, are guaranteed. The PSPT and LCT strategy minimize the path delay and the path cost of the multicast trees, respectively. Simulation results demonstrate that the performance benefits of the proposed ODQMRP in terms of the end-to-end tree delay, the tree cost, and the failure ratio of multicasting connections by comparison with the conventional non-QoS shortest path tree (SPT) strategy.
\end{abstract}

Index Terms-Satellite networks, multicast routing, quality of service, low earth orbit (LEO), highly elliptical orbit (HEO), geostationary earth orbit (GEO).

\section{INTRODUCTION}

$S^{A}$ ATELLITE networks have a wide range of potential applications in data communications and the Internet, mobile and personal communications, voice and telephony networks, broadcast and multicast of digital content, and so on [1]. There is no doubt that satellite networks will be an integral part of the newly emerging Next Generation Networks (NGN) [2] and the evolution of Future Networks (FN) [3], and also play a critical role in realizing the "global village" concept of the world [4]. The impetus to the NGN, even the revolutionary FN, which satellite networks provide can be summarized as follows [4]-[6].

Manuscript received February 15, 2011; revised May 15, 2011; accepted June 15, 2011
- Global connectivity anywhere and anytime.

- Cost-effective broadcast/multipoint services.

- World-wide direct and ubiquitous access to diversified environments, even remote, inaccessible areas.

- Connectivity in geographical areas where the terrestrial infrastructure has been damaged.

- Very flexible bandwidth-on-demand capabilities.

- Alternative channels for connections for which the bandwidth demands and traffic characteristics are unpredictable.

- Flexible network configuration and capacity allocation

- On-demand multimedia (integrated voice, data, and video) communications, such as distance learning, distributed software updates, telemedicine, and electronic commerce, etc.

Due to the rapidly and regularly changing network topology caused by the high mobility of satellites [7], routing in satellite networks faces great challenges. Previous routing schemes for ATM or ATM-type switches on-board satellites are designed based on the connection-oriented mechanisms that satellite networks own. The integration of the concept of virtual path connections (VPC) and a modified traditional routing scheme is introduced in [8], [9] to tackle the time-variant topology. In [10], a Finite State Automation (FSA) is used to model the Low Earth Orbit (LEO) satellite networks, and the routing problem is treated as a set of link assignment problems using a combinatorial optimization method. The handover rerouting protocol is presented in [11] to maintain the optimality of the initial route without performing a routing algorithm after intersatellite handovers. In [12], the probabilistic routing protocol (PRP) is investigated to reduce the number of rerouting attempts for the dynamic network topology.

However, with the great popularity of the Internet and the rapid development of the NGN in terrestrial networks, satellite networks will be required to provide connectionless service and transport IP-based traffic. Routing strategies for IP or IP-like switches on-board satellites have also been extensively studied. The 
DARTING algorithm [13] is devised to gear the periodic exchange of topology update messages until there is demand of delivering data messages. Nevertheless, the performance evaluation in [14] demonstrates that the DARTING algorithm requires a much higher overhead and has higher instability at network update periods. In [15], based on the geographic-based addresses, a distributed routing protocol is proposed to direct satellites to route packets in the direction that most reduces the remaining distance to the destination. The datagram routing algorithm (DRA) in [16], using the concept of logical locations of the LEO satellites, is introduced to forward the packets with the minimum propagation delay. In [17], the Multi-Layered Satellite Routing (MLSR) algorithm calculates routing tables efficiently using the collected delay measurements periodically. The Satellite Grouping \& Routing Protocol (SGRP) [18] forwards the packets on minimum delay paths regardless of the satellite mobility, and distributes the routing table calculation for LEO satellites to multiple Medium Earth Orbit (MEO) satellites.

With the explosive growth of Internet-based multimedia applications, such as push media, file distribution and caching, multimedia conferencing, multiplayer games, chat groups, and so on [19], multicasting constitutes an important service to perform the simultaneous distribution of the same multicasting packets from a single source node to a group of destinations in the satellite IP networks. Multicast routing is one of the key technologies in the multicasting service for satellite networks. In recent years, many conventional multicast routing protocols for terrestrial networks have been proposed [20]-[22] and effectively employed. In terms of the conditions of networks, the multicast routing protocols can be categorized into two types: a) "wired" multicast routing protocols; such as the Distance Vector Multicast Routing Protocol (DVMRP) [23], the Core Based Tree (CBT) [24], the Internet Group Management Protocol (IGMP) [25], the Multicast Extensions for OSPF (MOSPF) [26], etc; b) wireless multicast routing protocols, such as the Multicast Ad hoc On-Demand Distance Vector (MAODV) [27], the Associativity-Based Ad hoc Multicast (ABAM) [28], the On-Demand Multicast Routing Protocol (ODMRP) [29], the CoreAssisted Mesh Protocol (CAMP) [30], etc. However, these existing multicast routing protocols can not be very well suited for satellite IP networks.

At present, only a few multicast routing schemes in the literature have been developed for satellite IP networks. In [31], using the DRA [16] to create the multicast trees, a multicast routing algorithm for LEO satellite IP networks is introduced, which minimizes the end-to-end delay for real time multimedia services. The bandwidthefficient multicast routing mechanism [32] based on rectilinear Steiner trees for LEO satellite IP networks minimizes the total bandwidth and gains the limited overhead. Two multicast routing algorithms based on the dynamic approximate center (DAC) core selection method, i.e., the core-cluster combination-based shared tree (CCST) algorithm and the weighted CCST algorithm, are presented in [33]. The former significantly decreases the average tree cost, and the latter reduces the average end-to-end propagation delay. The distributed multicast routing protocol in [34] aims to minimize the total cost of the multicast trees in multi-layered satellite IP networks, including Geostationary Earth Orbit (GEO), MEO, and LEO layers. On the whole, the multicast routing schemes proposed for satellite IP networks in the literature can be divided into two categories: a) multicast routing for single-layered satellite IP networks; b) multicast routing for multi-layered satellite IP networks.

In addition, the future media rich applications such as media streaming, content delivery distribution and real time broadband access require satellite networks that inherently offer greater bandwidth and user level quality of service (QoS) guarantees [35]. In this regard, one of the challenges for multicasting communications in satellite networks is to design the QoS multicast routing protocols. QoS-aware multicast routing aims to find a multicast tree rooted from the source node, which not only spans to all the destination nodes, but also meet the QoS requirements. To our knowledge, some QoS unicast routing schemes for satellite networks have been proposed, such as the hierarchical \& distributed QoS routing protocol (HDRP) [36], the distributed QoS routing [37], the AntNet-based multi-QoS routing [38], the Predictive Routing Protocol (PRP) [39], etc. However, there is no QoS multicast routing protocol so far specifically developed for satellite IP networks.

In general, a combination of different layers of satellite constellations, such as GEO, LEO, MEO, and Highly Elliptical Orbit (HEO) satellite constellations, to build up a solid satellite network with multiple layers, can yield a much better performance than these layers individually [17], e.g., higher efficiency in the spectrum usage, flexible user's access and networking configuration, larger transmission capacity, strong invulnerability. Currently, the multi-layered satellite networks mainly make use of the combinations of different layers of GEO, LEO, MEO satellite constellations, namely, a) the double-layered satellite networks including the LEO/MEO architecture [8], [18], [40], and the LEO/LEO architecture [41], b) the conventional LEO/MEO/GEO architecture [17], [34]. However, the existing multilayered satellite networks can not provide the coverage over the special regions or the areas of high latitudes. In this paper, for the sake of better "global coverage", we take into account the demand of satellite communications over the special regions, e.g., the high-latitude areas, and present a triple-layered LEO/HEO/GEO satellite network architecture. On the basis of the novel hierarchical satellite network architecture, we adopt the concept of logical locations to isolate the mobility of LEO and HEO satellites and propose an on-demand QoS multicast routing protocol (ODQMRP) for satellite IP networks. In ODQMRP, the link state information piggybacked by each satellite is exchanged through the link state report process, and the network topology is acquired by the source node through the route discovery and route reply process. Furthermore, we introduce two strategies to 
create the multicast trees under the condition that the QoS requirements are guaranteed, i.e., the parallel shortest path tree (PSPT) strategy and the least cost tree (LCT) strategy. The PSPT and LCT strategy minimize the path delay and the path cost of the multicast trees, respectively. We also have evaluated the performance of our proposed ODQMRP under different strategies, i.e., PSPT and LCT, via computer simulations. Simulation results demonstrate that the performance benefits of ODQMRP in terms of the end-to-end tree delay, the tree cost, and the failure ratio of multicasting connections in contrast with the conventional shortest path tree (SPT) strategy.

The remainder of the paper is organized as follows. Section II introduces the triple-layered LEO/HEO/GEO satellite network architecture. In Section III, we present the formulation of the problem, and a description of ODQMRP is proposed in detail in Section IV. Section V evaluates the performance of our proposed ODQMRP. We conclude the paper in Section VI.

\section{TRIPLE-Layered SATEllite Network ARCHITECTURE}

The triple-layered satellite network architecture consists of a GEO constellation, several LEO and HEO constellations, and some fixed terrestrial gateways. The terrestrial gateways are in the coverage areas of GEO and LEO satellites and assumed to be the sources and destinations of multicasting communications and provide the interconnection to other ground wired/wireless networks. The circular coverage area on the Earth surface, i.e., the footprint of a single satellite, is the union of the cell-like areas covered by the spot beams of that satellite. The proposed satellite network architecture is illustrated in Fig. 1.

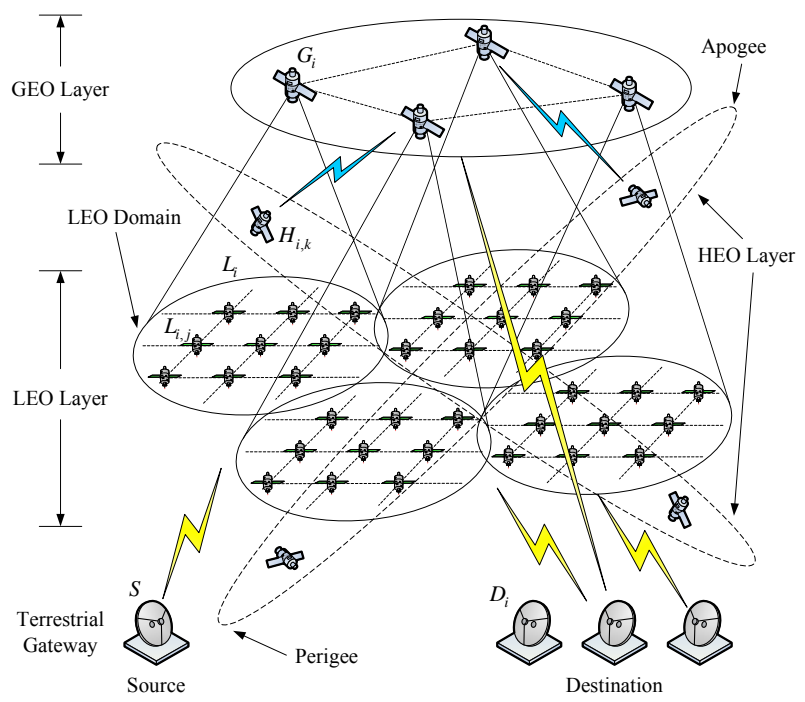

Figure 1. Triple-layered LEO/HEO/GEO satellite network architecture.

\section{A. Satellite Layers and Links}

The hierarchical satellite network architecture is divided into three layers in terms of the corresponding constellations.
1) GEO Layer: The GEO layer is composed of a GEO constellation which achieves the full coverage in the region of equator. The GEO satellites within a GEO constellation orbit the Earth at an altitude of about 36000 $\mathrm{km}$ and the angular velocity matches the Earth's rate of rotation. Assume that the total number of GEO satellites is $N_{G}$ and a GEO satellite is denoted by $G_{i}, i=1, \cdots, N_{G}$.

2) LEO Layer: The LEO layer is composed of several LEO constellations which cover the entire globe. The LEO satellites within a LEO constellation move with high velocities at altitudes typically with the range from $500 \mathrm{~km}$ to $1500 \mathrm{~km}$ above the surface of the Earth. Note that the LEO satellites in different LEO constellations have the same orbital altitude. Assume that the total number of satellites in LEO layer is $N_{L}$ and a LEO satellite is denoted by $L_{i, j}$, which is in the coverage area of the GEO satellite $G_{i}$. The logical location concept [16] is used to resolve the problems caused by the mobility of LEO satellites. Assume that Walker star pattern constellation [42] is applied in the LEO layer to organize the LEO satellites.

3) HEO Layer: The HEO constellations are introduced to provide coverage to selected areas of the globe, e.g. Earth's polar regions, over which most GEO satellites lack. The HEO layer is composed of all HEO satellites in the satellite network. The HEO satellites within a HEO constellation have an orbit elliptical in shape with the perigee altitude approaching $500 \mathrm{~km}$ and the apogee altitude about $50000 \mathrm{~km}$ above the ground. The satellite period varies from 8 to 24 hours. Assume that the total number of satellites in HEO layer is $N_{H}$ and a HEO satellite is denoted by $H_{i, k}$, which is in the coverage area of the GEO satellite $G_{i}$.

Three types of duplex links are maintained in the network. Satellites are connected to each other within the same layer via Inter-Satellite Links (ISLs), while the communication between satellites (e.g. GEO and LEO satellites) in different layers is completed via InterOrbital Links (IOLs). Note that coverage for communication services from HEO satellites is only provided when HEO satellites are moving very slowly relative to the globe while in the vicinity of apogee. For that reason, assume that the communication between GEO satellites and HEO satellites is accomplished via IOLs when HEO satellites are moving near apogee, while the communication between HEO satellites cannot be maintained through ISLs in our architecture. The terrestrial gateways can be directly connected to LEO satellites and GEO satellites via User Data Links (UDLs).

\section{B. Satellite Domains}

Taking the logical locations of satellites into account, we introduce satellite domains to organize the satellites in a hierarchical manner in order to isolate the mobility of satellites from upper layer, i.e., GEO layer.

1) LEO Satellite Domains: A LEO satellite domain $L_{i}$ is the set of logical locations of LEO satellites that are within the coverage of a GEO satellite $G_{i}$. This GEO satellite $G_{i}$ can just communicate with the LEO satellites through IOLs within a LEO satellite domain that is in the 
coverage of $G_{i}$. Furthermore, the GEO satellite $G_{i}$ is called the manager of the LEO satellite domain $L_{i}$. In terms of LEO layer, ISLs can be categorized into two types, i.e., intra-domain ISLs and inter-domain ISLs. Note that the LEO satellites are connected to their adjacent neighbors over the grid points in the same layer via intra-domain ISLs. Here, $L_{i}=\left\{L_{i, j} \mid j=1, \cdots, \mathcal{S}\left(L_{i}\right)\right\}$, where $\mathcal{S}(\cdot)$ is a size function that generates the total number of all satellites in a satellite domain.

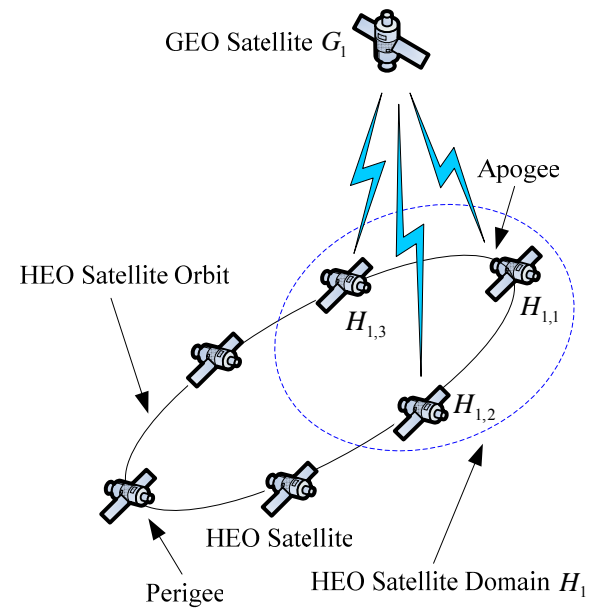

Figure 2. A partial view of a HEO satellite domain.

2) HEO Satellite Domains: A HEO satellite domain $H_{i}$ is the set of logical locations of HEO satellites that are within the coverage of a GEO satellite $G_{i}$. Note that different GEO satellites may have the same HEO satellite domains. For that reason, all the satellites in a HEO satellite domain have the IOLs with a certain number of GEO satellites that cover the same HEO satellite domain. The GEO satellite $G_{i}$ is also called the manager of the HEO satellite domain $H_{i}$. Here, $H_{i}=\left\{H_{i, k} \mid k=1, \cdots, \mathcal{S}\left(H_{i}\right)\right\}$. Assume that half of the HEO satellites within a HEO constellation in the same orbit have IOLs with a GEO satellite at time instant. A partial view of a HEO satellite domain is depicted in Fig. 2, where a GEO satellite $G_{1}$, a HEO constellation and a HEO satellite domain $H_{1}$ containing three HEO satellites, i.e., $H_{1,1}, H_{1,2}, H_{1,3}$, are illustrated.

\section{PROBLEM Formulation}

\section{A. Definitions}

Definition 1. The topology of satellite network based on our architecture is modeled as a connected directed graph $G=(V, E)$, where $V$ is the set of nodes representing the satellites and terrestrial gateways in our architecture and $E \subseteq V \times V$ is the set of links connecting the nodes, i.e., ISLs, IOLs and UDLs.

Definition 2. Let the terrestrial gateway $S \in V$ denote a source node of a multicasting communication, and other terrestrial gateways constitute a non-empty finite set of destination nodes, i.e., $D \subseteq V-\{S\}$, called a multicast group. A multicast tree $T=\left(V_{T}, E_{T}\right)$, for $V_{T} \subseteq V$ and
$E_{T} \subseteq E$, is a subtree of the graph $G=(V, E)$ rooted from $S$, which includes all of the nodes of $D$ and an arbitrary subset of $V-D$.

Definition 3. The link state of a link $l$ is composed of delay $\mathcal{D}(l)$, available bandwidth $\mathcal{B}(l)$ and cost $\mathcal{C}(l)$, for $l \in E \quad, \quad$ where $\quad \mathcal{D}(l): E \rightarrow \boldsymbol{R}^{+} \quad, \quad \mathcal{B}(l): E \rightarrow \boldsymbol{R}^{+} \quad$ and $\mathcal{C}(l): E \rightarrow \boldsymbol{R}^{+}$are delay function, available bandwidth function and cost function, respectively. Note that the delay $\mathcal{D}(l)$ of a link $l$ contains three delay components: a) radio propagation delay, b) queuing delay, and c) protocol processing delay.

Definition 4. The available path bandwidth $B(P)$ of a path $P$ is the minimum bandwidth of the links along the path, i.e.,

$$
B(P)=\arg \min \left\{\mathcal{B}\left(l_{i}\right) \mid l_{i} \in P, i=1, \cdots, \mathcal{P} \mathcal{L}(P)\right\}
$$

where $\mathcal{P} \mathcal{L}(\cdot)$ is a function that returns the number of links in the path $P$.

Definition 5. The available tree bandwidth $B(T)$ of a multicast tree $T$ is the minimum bandwidth of the links in the multicast tree, i.e.,

$$
B(T)=\arg \min \left\{\mathcal{B}\left(l_{i}\right) \mid l_{i} \in T, i=1, \cdots, \mathcal{T L}(T)\right\}
$$

where $\mathcal{T L}(\cdot)$ is a function that returns the number of links in the tree $T$.

Definition 6. The path delay $D(P)$ of a path $P$ is the sum of the delay of the links on the path, i.e.,

$$
D(P)=\sum_{i=1}^{\mathcal{P} \mathcal{L}(P)} \mathcal{D}\left(l_{i}\right)
$$

Definition 7. The tree delay $D(T)$ of a multicast tree $T$ is the maximum delay of the paths from source node $S$ to the destination nodes of $D$ on the multicast tree, i.e.,

$$
D(T)=\arg \max \left\{D\left(P_{S \rightarrow D_{i}}\right)|i=1, \cdots,| D \mid\right\}
$$

where $P_{S \rightarrow D_{i}}$ denotes a feasible path from the source node $S$ to destination node $D_{i}$, and $|D|$ denotes the number of destination nodes.

Definition 8. The path cost $C(P)$ of a path $P$ is defined as the product of the available path bandwidth and the path delay of the path $P$, i.e.,

$$
C(P)=B(P) \times D(P)
$$

Definition 9. The least cost path $P_{A \rightarrow B}^{*}$ from node $A$ to node $B$ is defined as a path that satisfies

$$
C\left(P_{A \rightarrow B}^{*}\right)=\arg \min \left\{C\left(P_{A \rightarrow B}^{i}\right) \mid i=1, \cdots, \mathcal{P N}\left(P_{A \rightarrow B}\right)\right\}
$$

where $P_{A \rightarrow B}$ denotes a feasible path from the node $A$ to the node $B$, and $\mathcal{P N}(\cdot)$ is a function that returns the number of feasible paths from the node $A$ to the node $B$.

Definition 10. The tree cost $C(T)$ of a multicast tree $T$ is defined as the product of the available tree bandwidth and the tree delay of the multicast tree $T$, i.e., 


$$
C(T)=B(T) \times D(T)
$$

\section{B. Problem Statement}

Our problem is: given a satellite network $G=(V, E)$, a source node $S$, a multicast group $D$, a delay bound $\Delta$ and a bandwidth bound $\Omega$, respectively, to construct a multicast tree $T=\left(V_{T}, E_{T}\right)$ which spans $S$ and $D$ such that the tree cost defined in (7) is minimized under the condition that the accumulated available tree bandwidth and tree delay of the multicast tree $T$ satisfy the following required QoS constraints

- Delay constraint: $D(T) \leq \Delta$;

- Bandwidth constraint: $B(T) \geq \Omega$.

\section{On-Demand QoS Multicast Routing Protocol}

Our proposed on-demand QoS multicast routing protocol (ODQMRP) is mainly composed of five parts, namely, link state report, route discovery, route reply, route maintenance and multicast tree creation. We now discuss the operation of the proposed protocol in detail.

\section{A. Link State Report}

In the link state report process, the available bandwidth and the delay of each link $l \in E$ in the satellite network $G(V, E)$ are established and the related link state information is recorded in each node. The link state report process is initiated whenever a source node (i.e., a source terrestrial gateway) receives a QoS request from the application layer for setting up a multicasting connection with a multicast group $D$ and the given delay and bandwidth bound constraints, i.e., $\Delta$ and $\Omega$. The source terrestrial gateway initially generates a report request (REPORT_REQ) message and then transmits the REPORT_REQ message to a GEO satellite via a UDL. When receiving the REPORT_REQ message, the GEO satellite follows the steps below to complete the link state report process.

1) Link State Report Request: The link state report request process can be described as follows.

(a) The GEO satellite forwards the REPORT_REQ message to other adjacent GEO satellites (i.e., the neighbors of the GEO satellite) via ISLs.

(b) When the REPORT_REQ message are received by all the GEO satellites in the GEO layer, each GEO satellite sends the REPORT_REQ message to the LEO and HEO satellites within its covered LEO satellite domain and HEO satellite domain through IOLs.

(c) In the LEO layer, the LEO satellite floods the REPORT_REQ message to other LEO satellites within the same LEO satellite domain via intradomain ISLs and across different domains via inter-domain ISLs.

(d) The members of the multicast group $D$ (i.e., $|D|$ destination terrestrial gateways) receive the REPORT_REQ message from the GEO and LEO satellites via UDLs.

2) Link State Interaction: After all the nodes in the satellite network acquire the REPORT_REQ message, the link state interaction process is initiated. The link state interaction process can be described as follows.

(a) The members of the multicast group $D$ transmit a state report (STATE_REPORT) message to the GEO/LEO satellites through the reverse UDLs. The format of the STATE_REPORT message is illustrated in Fig. 3. The "Type" refers to the message type and is set to 1 for the STATE_REPORT message. The fields "Available Bandwidth" and "Delay" record the available bandwidth and the delay between a node and its downstream node over the corresponding link. The pair < Node Address, State Report Sequence Number $>$ uniquely identifies the STATE_REPORT message. The "State Report Sequence Number" is monotonically incremented whenever a node issues a new STATE_REPORT message to its downstream node and can be used to check the duplicate copies of an old STATE REPORT message for the downstream node. In other words, when a downstream node receives a STATE_REPORT message, if it has already received a STATE_REPORT message with the same "Node Address" and "State Report Sequence Number", it drops the redundant STATE_REPORT message in order to reduce the communication load. The "Downstream Node Address" identifies the downstream node, and the "Reserved" is set to 0 for ignoring and non-zero for receiving. When receiving the STATE REPORT messages from the destination terrestrial gateways, the GEO and LEO satellites acquire the link state information, i.e., the available bandwidth and the delay between the GEO satellites or LEO satellites and the destination terrestrial gateways.

\begin{tabular}{|c|c|c|c|}
\hline Type & Available Bandwidth & Delay & Reserved \\
\hline \multicolumn{4}{|c|}{ State Report Sequence Number } \\
\hline \multicolumn{4}{|c|}{ Node Address } \\
\hline \multicolumn{4}{|c|}{ Downstream Node Address } \\
\hline
\end{tabular}

Figure 3. The STATE_REPORT message format.

(b) In the LEO layer, the LEO satellite receives the STATE_REPORT message from the upstream node (i.e., the destination terrestrial gateway) and then issues its STATE_REPORT message to other LEO satellites within the same LEO satellite domain via intra-domain ISLs and across different domains via inter-domain ISLs.

(c) The LEO satellites in the same LEO satellite domain transmit their STATE_REPORT messages via IOLs to their manager, the GEO satellite.

(d) In the GEO layer, the GEO satellite sends its STATE_REPORT messages to other adjacent GEO satellites via ISLs. When exchanging their link state information, the GEO satellite delivers 
the corresponding STATE_REPORT messages to the HEO satellites within its covered HEO satellite domain through IOLs and also to the source terrestrial gateway through UDLs.

\section{B. Route Discovery}

Our proposed QoS multicast routing protocol is an "on demand". The nodes neither maintain any routing information nor participate in any periodic routing table exchanges when there is no QoS multicast routing call received by the multicast source.

The route discovery is initiated by the source terrestrial gateway when the link state report process is completed. According to the QoS multicast routing request, the source node initiates the route discovery by flooding a route request (RREQ) message to its neighbor nodes. The RREQ message inherits the modified format of RREQ message in traditional Ad hoc On-Demand Distance Vector (AODV) routing protocol [43] and the format of the RREQ message is depicted in Fig. 4(a). The "Type" refers to the message type and is set to 2 for the RREQ message. The fields " $\Omega$ " and " $\Delta$ " denote the given bandwidth bound and delay bound, respectively and prevent unnecessary network-wide dissemination of RREQ messages. The pair < Source Address, RREQ ID $>$ uniquely identifies the RREQ message. The "RREQ ID" is monotonically increasing whenever the source node issues a new RREQ message to its neighbor nodes and can be used to check the duplicate copies of an old RREQ message for the neighbor nodes. The "Multicast Group Address List" indicates the set of destination nodes and the "Path" records the routing information. The "Accumulated Delay" records the sum of delay along the path. Furthermore, the "Reserved" has the same meaning with that in the STATE_REPORT message.

\begin{tabular}{|c|c|c|c|}
\hline Type & $\Omega$ & $\Delta$ & Reserved \\
\hline \multicolumn{3}{|c|}{ RREQ ID } \\
\hline \multicolumn{3}{|c|}{ Multicast Group Address List } \\
\hline \multicolumn{3}{|c|}{ Source Address } \\
\hline \multicolumn{3}{|c|}{ Path } \\
\hline \multicolumn{3}{|c|}{ Accumulated Delay } \\
\hline
\end{tabular}

(a) RREQ message format

\begin{tabular}{|c|c|c|c|}
\hline Type & $\Omega$ & $\Delta$ & Reserved \\
\hline \multicolumn{3}{|c|}{ RREP ID } \\
\hline Destination Address \\
\hline Source Address \\
\hline Path Set \\
\hline Lifetime \\
\hline
\end{tabular}

(b) RREP message format
Figure 4. The RREQ and RREP message format.

When an intermediate node receives a RREQ message, it checks two items to decide whether to reflood the newly received RREQ message.

(a) Whether there is enough available bandwidth $\mathcal{B}\left(l^{\prime}\right)$ over the link $l^{\prime}$ between the last hop node and itself according to the link state information, i.e., whether there exists $\mathcal{B}\left(I^{\prime}\right) \geq \Omega$. If $\mathcal{B}\left(l^{\prime}\right)<\Omega$, it means that there is no available bandwidth to meet the QoS requirements for establishing the connection through that link and the node drops the RREQ message.

(b) Whether the sum of the value of the "Accumulated Delay" and the delay $\mathcal{D}\left(l^{\prime}\right)$ over the link $l^{\prime}$ meets the delay bound constraint, i.e., $A D+\mathcal{D}\left(l^{\prime}\right) \leq \Delta$, where $A D$ denotes the value in the field "Accumulated Delay". If $A D+\mathcal{D}\left(l^{\prime}\right)>\Delta$, it means that the delay requirement cannot be guaranteed and the node drops the RREQ message.

Otherwise, if the RREQ message is received by the node for the first time, the node enters its own address to the field "Path" and inputs the value of $\left(A D+\mathcal{D}\left(l^{\prime}\right)\right)$ into the field "Accumulated Delay", and then disseminates the RREQ message out. Note that this intermediate node is also called the forwarding node. If the newly received RREQ message with the pair < Source Address, RREQ ID > was received before, it means that there exists another path from the source to the node. The node records this path information and discards the RREQ message.

\section{Route Reply}

This operation in the route discovery process will be repeated node by node until the delay bound or available bandwidth bound cannot be guaranteed. Eventually, a RREQ message will arrive at a destination terrestrial gateway and the destination node will also check two items described in the route discovery process to determine whether the QoS constraints are satisfied. If so, the destination node will wait for a certain timeout $T_{1}$ to receive multiple copies of the RREQ messages. Note that each copy indicates a possible path. Then the destination node creates a route reply (RREP) message including all the information about the multiple possible paths reaching it and sends the RREP message back to the source node. Meanwhile, the destination node can also act as an intermediate node and continues to forward the RREQ message until the QoS constraints are not guaranteed.

The format of the RREP message is shown in Fig. 4(b). The "Type" refers to the message type and is set to 3 for the RREP message. The pair < Destination Address, RREP ID > uniquely identifies the RREP message and the "RREP ID" is monotonically increasing whenever the destination node sends a new RREP message back to the source node. The "Destination Address" is the address of the destination node that has received the RREQ message. The "Lifetime" denotes a value of a pre-defined timeout $\mathrm{T}_{2}$ for which the nodes receiving the RREP message consider the route to be valid, and the "Path Set" is the set of multiple possible paths from the source node to the destination node. In the field "Path Set", each path is marked with the information of accumulated delay and available bandwidth from the source node to the destination node. The values of other entries in the RREP message are consistent with the corresponding entries in the RREQ message.

After a pre-defined timeout $T_{2}$, i.e., the value of the "Lifetime" field, the source node does not receive any more RREP messages and the route discovery and route reply process terminate. When the source node receives all the RREP messages, it gets a partial topology from it 
to the multicast group in the satellite network $G=(V, E)$. Figure 5 gives an example of a partial topology generated of the satellite network by the route discovery and route reply process, where the nodes in the partial topology are denoted by the corresponding notations described in our architecture, and the given delay bound is set to $\Delta=1200 \mathrm{~ms}$ and bandwidth bound is set to $\Omega=100 \mathrm{Mb} / \mathrm{s}$. The integer parameters along the links are represented as (delay, available bandwidth), where the units of the parameters are $\mathrm{ms}$ and $\mathrm{Mb} / \mathrm{s}$, respectively. Our multicast tree creation strategies are dependent on this partial topology and the global topology of the satellite network is not necessary for the source node. Furthermore, the source node may have multiple parallel paths to some destination nodes, e.g., the two possible paths in Fig. 5 shown as follows.

$$
\left\{\begin{array}{l}
S \rightarrow L_{4,3} \rightarrow L_{4,4} \rightarrow G_{4} \rightarrow G_{3} \rightarrow L_{3,9} \rightarrow D_{3} \\
S \rightarrow L_{1,2} \rightarrow L_{1,5} \rightarrow G_{1} \rightarrow G_{2} \rightarrow L_{2,5} \rightarrow L_{2,4} \rightarrow L_{3,8} \rightarrow L_{3,5} \rightarrow D_{3} \text { path 2 }
\end{array}\right.
$$

where the delay and available bandwidth of path 1 are $620 \mathrm{~ms}$ and $120 \mathrm{Mb} / \mathrm{s}$, respectively, and the delay and available bandwidth of path 2 are $817 \mathrm{~ms}$ and $100 \mathrm{Mb} / \mathrm{s}$, respectively. Obviously, as shown in Fig. 5, the destination node may also serve as a forwarding node, e.g., the path from $S$ to $D_{1}$ shown as follows.

$$
\text { path } 1 \rightarrow L_{3,6} \rightarrow L_{2,3} \rightarrow L_{2,2} \rightarrow L_{3,1} \rightarrow D_{1} \quad \text { path } 3
$$

where the destination node $D_{3}$ in path 3 is the forwarding node, and the delay and available bandwidth of path 3 are $775 \mathrm{~ms}$ and $100 \mathrm{Mb} / \mathrm{s}$, respectively.

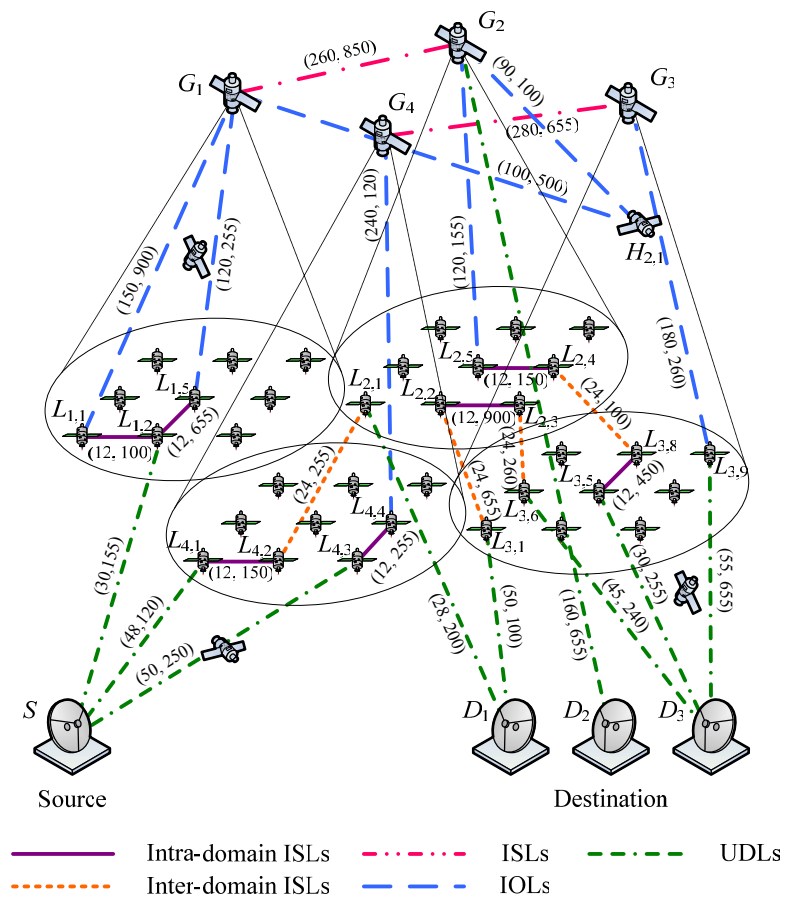

Figure 5. An example of a partial network topology generated by route discovery and route reply process.

\section{Route Maintenance}

1) Joining Multicast Group: The joining multicast group process is initiated whenever a new terrestrial gateway wants to join a multicast group. The new gateway firstly creates a STATE_REPORT message where the "Node Address" is filled with the gateway's address, i.e., the destination address, and then transmits the STATE_REPORT message to the GEO/LEO satellites through the UDLs. Through the link state interaction process, the nodes with the corresponding links in the satellite network acquire the link state information. Then the new gateway broadcasts a join request (JOIN_REQ) message with the format depicted in Fig. 6(a). The "Type" refers to the message type and is set to 4 for the JOIN_REQ message. The pair $<$ Terrestrial Gateway Āddress, JOIN_REQ ID > uniquely identifies the JOIN_REQ message and the "JOIN_REQ ID" is monotonically increasing whenever the new gateway node floods a new JOIN_REQ message to its neighbor nodes. The values of other entries in the JOIN_REQ message are consistent with the corresponding entries in the RREQ message.

When an intermediate node receives a JOIN_REQ message, it checks two items described in the route discovery process to decide whether to reflood the newly received JOIN_REQ message. The new gateway will send multiple JOIN_REQ messages out within a predefined timeout $T_{3}$ and the JOIN_REQ messages are flooded until they arrive at a desitination node of the multicast group. The destination node firstly will wait for a certain timeout $T_{4}$ to receive multiple copies of the JOIN_REQ messages. Secondly, according to the field "Path" information, it will check two items described in the route discovery process to determine whether the QoS constraints are satisfied. If so, the destination node creates a join reply (JOIN_REP) message including all the information about the multiple possible paths reaching it and sends the JOIN_REP message back to the new gateway. Note that this destination node serves as a forwarding node and the source node does not know the information about this new terrestrial gateway.

The format of the JOIN_REP message is shown in Fig. 6(b). The "Type" refers to the message type and is set to 5 for the JOIN_REP message. The pair $<$ Destination Address, JOIN_REP ID > uniquely identifies the JOIN_REP message and the "JOIN_REP ID" is monotonically increasing whenever the destination node sends a new JOIN_REP message back to the new gateway node. The values of other entries in the JOIN_REP message are consistent with the corresponding entries in the RREP message.

After a pre-defined timeout $T_{5}$, the new gateway node does not receive any more JOIN_REP messages and the joining multicast group process terminates. 


\begin{tabular}{|c|c|c|}
\hline Type & Path & Reserved \\
\hline \multicolumn{3}{|c|}{ JOIN_REQ ID } \\
\hline Terrestrial Gateway Address \\
\hline \multicolumn{2}{|c|}{ Accumulated Delay } \\
\hline
\end{tabular}

(a) JOIN REQ message format

\begin{tabular}{|c|c|c|c|}
\hline Type & $\Omega$ & $\Delta$ & Reserved \\
\hline \multicolumn{3}{|c|}{ JOIN_REP ID } \\
\hline \multicolumn{2}{|c|}{ Terrestrial Gateway Address } \\
\hline \multicolumn{3}{|c|}{ Destination Address } \\
\hline
\end{tabular}

(b) JOIN_REP message format
Figure 6. The JOIN_REQ and JOIN_REP message format.

2) Leaving Multicast Group: The leaving multicast group process is initiated whenever a destination terrestrial gateway wants to leave a multicast group. The destination terrestrial gateway firstly sends out multiple join negative acknowledgement (JOIN_NAK) messages to its neighbor nodes and then deletes all the routing information of the neighbor nodes. The format of the JOIN_NAK message is shown in Fig. 7. The pair $<$ Terrestrial Gateway Address, JOIN NAK Sequence Number $>$ uniquely identifies the JOIN_NAK message. The "Type" refers to the message type and is set to 6 for the JOIN_NAK message. The "JOIN_NAK Sequence Number" is monotonically incremented whenever the destination terrestrial gateway issues a new JOIN_NAK message to its neighbor node.

When receiving a JOIN_NAK message, a neighbor node checks whether it has an upstream node or a downstream node. If so, the neighbor node prunes the link from the destination terrestrial gateway and deletes the routing information of the destination terrestrial gateway, and then transmits the JOIN_NAK message out to notify that the destination terrestrial gateway has been leaving the multicast group. Otherwise, the neighbor node will check whether it is a member of the multicast group. If so, the neighbor node just prunes the link from the destination terrestrial gateway. Otherwise, the neighbor node becomes a non-forwarding node and withdraws from the QoS multicasting communications.

\begin{tabular}{|l|c|c|}
\hline Type & Path & Reserved \\
\hline \multicolumn{2}{|l|}{ JOIN_NAK Sequence Number } \\
\hline \multicolumn{2}{|c|}{ Terrestrial Gateway Address } \\
\hline
\end{tabular}

Figure 7. The JOIN_NAK message format.

\section{E. Multicast Tree Creation}

The multicast tree creation process is activated by the source node at the end of the route discovery and route reply process. As mentioned previously, the source node has maintained multiple parallel paths from itself to several destination nodes in the multicast group. Consequently, the main goal of the source node is to select one of the parallel paths to set up a connection, and then proceed to create a multicast tree. Here, we present two strategies to construct the multicast tree under the condition that the QoS requirements are guaranteed, namely, the parallel shortest path tree (PSPT) strategy and the least cost tree (LCT) strategy.

1) Parallel Shortest Path Tree Strategy: In the PSPT strategy, we will not apply the classic Dijkstra's algorithm
[44], i.e., the Shortest Path Tree (SPT), to find the path, and further to produce the multicast tree. However, we will employ the results of the route discovery and route reply process, i.e., the multiple parallel paths from the single source to the destination nodes, which are recorded in the field "Path Set" in the RREP message. Here, we consider that the PSPT possesses the shortest delay for the reason that each path from the source node to the destination node in the multicast group is a path with the shortest path delay.

The basic idea of the PSPT strategy works as follows. In the case of a received RREP message from a destination node $D_{i}, i=1, \cdots,|D|$, the source node initially checks whether the field "Path Set" contains multiple parallel paths. Assume that the "Path Set" from a destination node $D_{i}$ is denoted by $P S_{i}$. If so, according to the RREP message, the source node computes the path delay $D\left(P_{i, j}\right)$ of each path $P_{i, j}, j=1, \cdots,\left|P S_{i}\right|$, and then compares $D\left(P_{i, j}\right)$ to select a path $P_{i, j}^{*}$ with the shortest path delay, i.e.,

$$
D\left(P_{i, j}^{*}\right)=\arg \min \left\{D\left(P_{i, j}\right)|i=1, \cdots,| D|, j=1, \cdots,| P S_{i} \mid\right\}
$$

Therefore, the path $P_{i, j}^{*}$ is selected as a path form the source node to the destination node $D_{i}$ for setting up a multicasting connection. Note that the bandwidth constraint of the path $P_{i, j}^{*}$ is also guaranteed. If the field "Path Set" contains just one path, the source node employs this path to establish a connection. This operation will proceed until the source node acquires $|D|$ paths with the shortest path delay. Then the multicast tree is constructed and the source node starts the multicasting session.

2) Least Cost Tree Strategy: The PSPT strategy can bring the minimum path delay in the multicast tree, but not optimize the path cost in the multicast tree. The LCT strategy takes into consideration both of the QoS requirements, i.e., the delay bound $\Delta$ and the bandwidth bound $\Omega$, in order to reduce the path cost, further to optimaize the tree cost.

The basic idea of the LCT strategy works as follows. When receiving a RREP message from a destination node $D_{i}, i=1, \cdots,|D|$, the source node gets the information of accumulated delay and available bandwidth from the source node to this destination node along a path $P_{i, j}$, $j=1, \cdots,\left|P S_{i}\right|$, i.e., the path delay $D\left(P_{i, j}\right)$ and the available path bandwidth $B\left(P_{i, j}\right)$. Therefore, the source node can compute the path cost $C\left(P_{i, j}\right)=B\left(P_{i, j}\right) \times D\left(P_{i, j}\right)$ for the path $P_{i, j}$, and then compare $C\left(P_{i, j}\right)$ to select a path $P_{i, j}^{*}$ with the least path cost, i.e.,

$$
C\left(P_{i, j}^{*}\right)=\arg \min \left\{C\left(P_{i, j}\right)|i=1, \cdots,| D|, j=1, \cdots,| P S_{i} \mid\right\}
$$

After a pre-defined timeout $T_{2}$, the source node gains all the information about the paths with least path cost from itself to each destination node in the multicast group. 
Afterwards, the source node follows the steps below to create a multicast tree.

(a) Construct two node sets $K=\{S\} \bigcup D$ and $H_{0}=\{S\}$.

(b) Start with a subtree $T_{0}=\left(V_{0}, E_{0}\right)$, where $V_{0}=\{S\}$ and $E_{0}=\varnothing$.

(c) For $\alpha=1, \cdots,|D|$, the source node finds a node in $K-H_{\alpha-1}$, i.e., a destination node $D_{i}$, such that the path cost from the source node to $D_{i}$ is minimum among all the paths with the least path cost, namely, $D_{i}=\arg \min \left\{C\left(P_{i, j}^{*}\right)|i=1, \cdots| D,|, j=1, \cdots|, P S_{i} \mid\right\}$

Construct the subtree $T_{\alpha}=\left(V_{\alpha}, E_{\alpha}\right)$ by adding the path $P_{i, j}^{*}$ between the source node and $D_{i}$ to $T_{\alpha}$, i.e., set $\quad V_{\alpha}=V_{\alpha-1} \bigcup$ nodes in $P_{i, j}^{*}$ \} and $E_{\alpha}=E_{\alpha-1} \cup\left\{\right.$ links in $\left.P_{i, j}^{*}\right\} \quad$. Meanwhile, set $H_{\alpha}=H_{\alpha-1} \bigcup\left\{D_{i}\right\}$.

When the multicast tree is created, the source node starts the multicasting session.

\section{PERformance Evaluation}

In this section, we evaluate the performance of ODQMRP under different strategies, i.e., PSPT and LCT, by comparing them with the conventional non-QoS SPT [44] strategy via computer simulations using STK 6.0 and NS-2.

In our empirical study, three performance metrics, i.e., a) the end-to-end tree delay, b) the tree cost, and c) the failure ratio of multicasting connections, are used to evaluate the performance of the proposed ODQMRP with PSPT (denoted by ODQMRP-PSPT), ODQMRP with LCT (denoted by ODQMRP-LCT), and the traditional SPT strategy.

\section{A. Simulation Setup}

In our simulations, the constellation parameters of the triple-layer satellite network are given in Table I. The performance of coverage from the proposed triple-layer satellite network is illustrated in Fig. 8. According to Fig. 8 , the proposed triple-layered satellite network can offer coverage over the areas varying from $75^{\circ} \mathrm{S}$ to $90^{\circ} \mathrm{N}$ with 24 hour uninterrupted. We use the non-uniform distribution [34] to determine the positions of the terrestrial gateways, including the source node and the multicast group. Moreover, we assume that the capacity of all ISLs, IOLs, and UDLs are set to $655 \mathrm{Mb} / \mathrm{s}$, each outgoing link has a buffer space of $20 \mathrm{MB}$.

In order to describe the QoS requirements for different application services, we present three different QoS application types, namely, the QoS classes, representing three multicasting scenarios. The multicasting sessions are assigned randomly to one of these QoS classes defined in Table II.

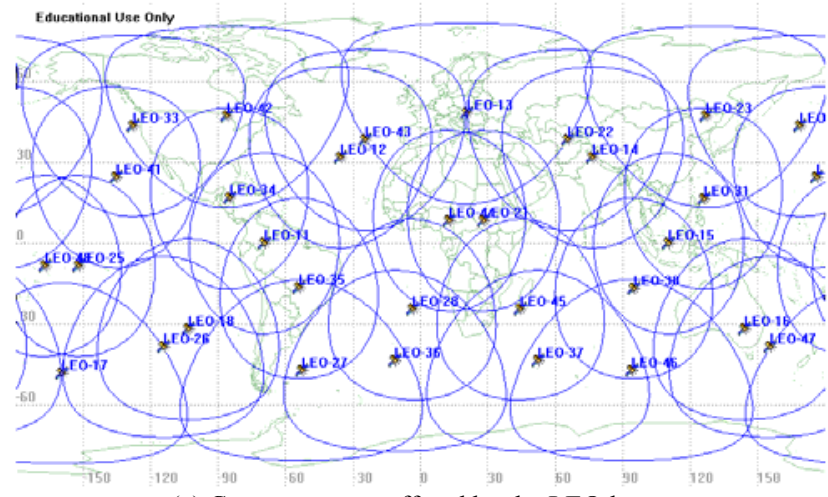

(a) Coverage areas offered by the LEO layer

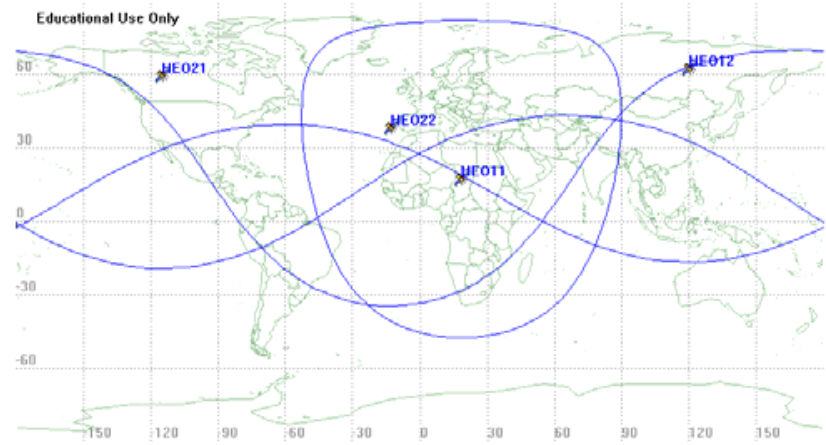

(b) Coverage areas offered by the HEO layer

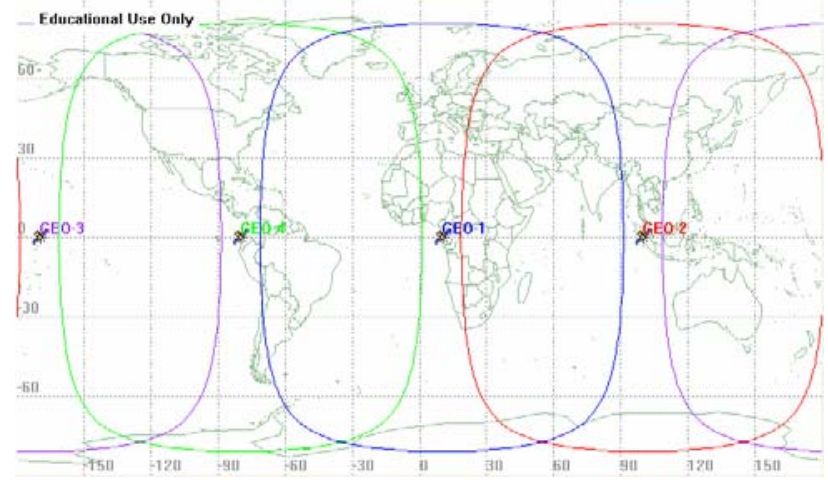

(c) Coverage areas offered by the GEO layer

Figure 8. Illustration of near-global coverage from the satellite network using the proposed triple-layered LEO/HEO/GEO architecture.

TABLE I. PARAMETERS FOR TRIPLE-LAYERED SATEllite NETWORKS.

\begin{tabular}{|c|c|c|c|}
\hline Parameters & LEO Layer & HEO Layer & GEO Layer \\
\hline Type of orbit & $\begin{array}{c}\text { LEO } \\
\text { Recursive } \\
\text { orbit }\end{array}$ & $\begin{array}{c}\text { HEO } \\
\text { Recursive } \\
\text { orbit }\end{array}$ & GEO \\
\hline Altitude & $1262 \mathrm{~km}$ & $\begin{array}{l}27000 \mathrm{~km}(\mathrm{~A}) \\
800 \mathrm{~km}(\mathrm{P})\end{array}$ & $35786 \mathrm{~km}$ \\
\hline Orbital period & $6628 s$ & $8 \mathrm{~h}$ & $24 \mathrm{~h}$ \\
\hline Number of satellites & 32 & 4 & 4 \\
\hline $\begin{array}{l}\text { Number of orbital } \\
\text { planes }\end{array}$ & 4 & 2 & 1 \\
\hline Orbit inclination angle & $48^{\circ}$ & $63.4^{\circ}$ & - \\
\hline $\begin{array}{l}\text { Minimum elevation } \\
\text { angle }\end{array}$ & - & $10^{\circ}$ & $5^{\circ}$ \\
\hline Constellation type & Walker star & Draim & - \\
\hline Semi-major axis & - & $20278 \mathrm{~km}$ & - \\
\hline Eccentricity & - & 0.646 & - \\
\hline Argument of perigee & 一 & $270^{\circ}$ & - \\
\hline Phase factor & 1 & - & 一 \\
\hline $\begin{array}{l}\text { Ascending node } \\
\text { longitude }\end{array}$ & - & $90^{\circ} \mathrm{E}$ & - \\
\hline
\end{tabular}


TABLE II. QOS CLASSES AND REQUIREMENTS

\begin{tabular}{clll}
\hline \hline QoS Classes & \multicolumn{1}{c}{$\begin{array}{c}\text { Delay } \\
\text { Requirements }\end{array}$} & $\begin{array}{c}\text { Available } \\
\text { Bandwidth } \\
\text { Requirements }\end{array}$ & $\begin{array}{c}\text { Application } \\
\text { Types }\end{array}$ \\
\hline Class 0 & $600 \mathrm{~ms}$ & $155 \mathrm{Mb} / \mathrm{s}$ & $\begin{array}{l}\text { High-speed data } \\
\text { on-demand }\end{array}$ \\
Class 1 & $400 \mathrm{~ms}$ & $256 \mathrm{Mb} / \mathrm{s}$ & $\begin{array}{l}\text { High-resolution } \\
\text { color images } \\
\text { Video } \\
\text { teleconferencing }\end{array}$ \\
\hline \hline
\end{tabular}

\section{B. Simulation Results and Analysis}

1) Performance Comparison of End-to-End Tree Delay: In the first set of experiments, we observe the endto-end tree delay of the SPT strategy and the proposed ODQMRP, and the multicast group size is set to 50 . Figure 9(a), (b), and (c) depict the performance of the end-to-end tree delay of the SPT strategy, the ODQMRPPSPT, and the ODQMRP-LCT, under the QoS Class 0, respectively. It can be easily seen that the end-to-end tree delay of the SPT strategy and the ODQMRP-PSPT vary a lot in the range of $0.2 \mathrm{~s}$ to $0.6 \mathrm{~s}$, with the increase of the simulation time. However, as the simulation time increases, the end-to-end tree delay of the ODQMRPLCT remains almostly steady with the range of $0.4 \mathrm{~s}$ to 0.6 s. Overall, since the ODQMRP-LCT mainly optimizes the tree cost, we observe that the end-to-end tree delay of the SPT strategy and the ODQMRP-PSPT are slightly smaller than that of the ODQMRP-LCT.

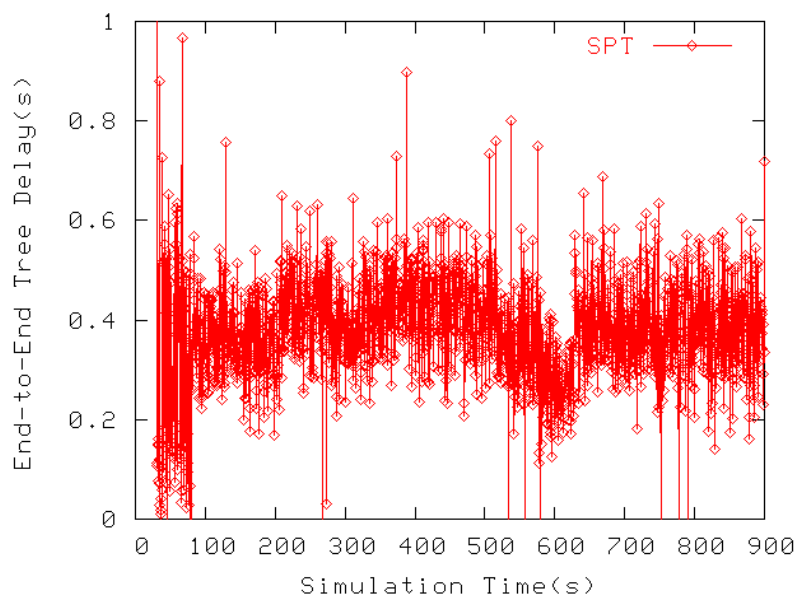

(a) The end-to-end tree delay of the SPT.

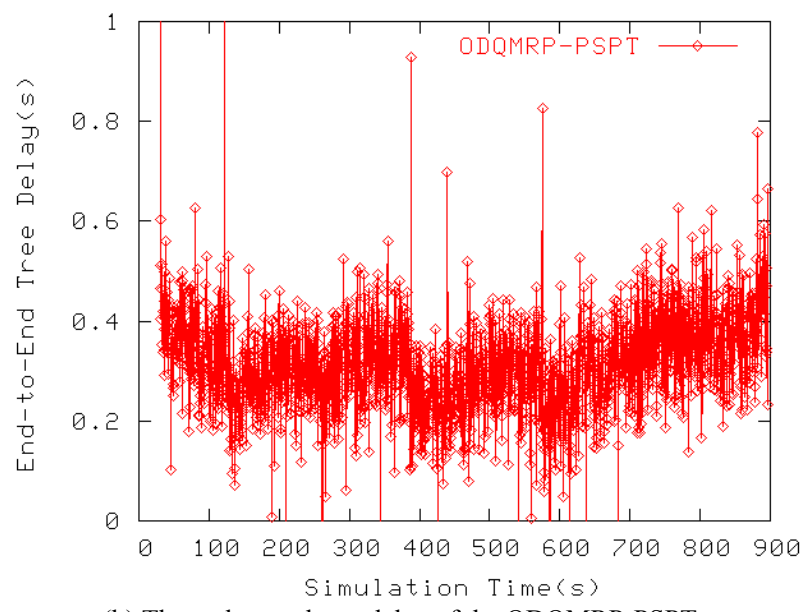

(b) The end-to-end tree delay of the ODQMRP-PSPT.

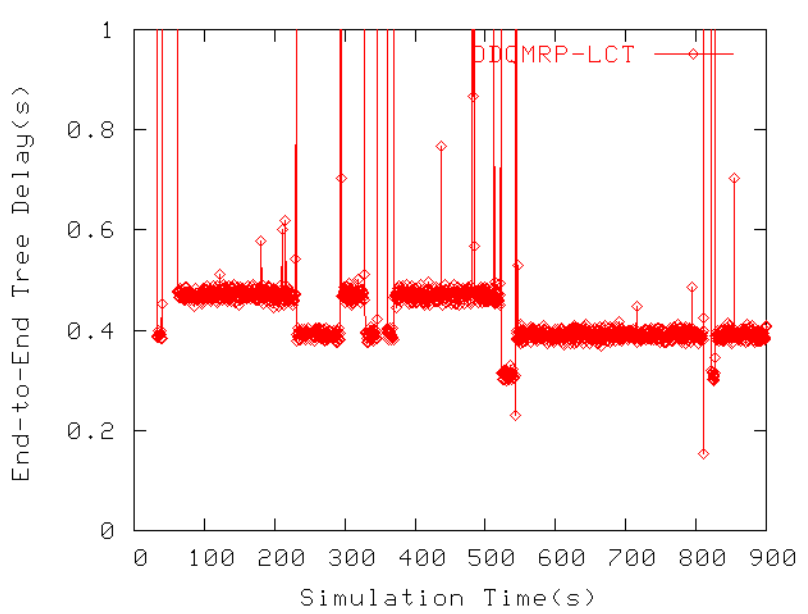

(c) The end-to-end tree delay of the ODQMRP-LCT.

Figure 9. Performance comparison of the end-to-end tree delay of the SPT strategy and the proposed ODQMRP under QoS Class 0.

2) Performance Comparison Under Different QoS Classes: In this set of experiments, we compare the performance of the SPT strategy, the ODQMRP-PSPT, and the ODQMRP-LCT, in terms of the performance metrics, i.e., the end-to-end tree delay, the tree cost, and the failure ratio.

Figure 10 shows the comparison of the end-to-end tree delay versus the multicast group size between the SPT strategy and the proposed ODQMRP for different QoS Classes. In Fig. 10(a), (b), and (c), we can obviously see that the end-to-end tree delay of the SPT strategy and the ODQMRP-PSPT are a little lower than that of the ODQMRP-LCT with the range of 10 to 30 of the multicast group size for the reason that the SPT strategy and the ODQMRP-PSPT aim at optimizing the end-toend tree delay. Furthermore, the SPT strategy finds the path based on the minimum path delay during the route discovery and reply, whereas the proposed ODQMRPPSPT constructs the multicast tree using the minimum tree delay after the process of the route discovery and reply.

Moreover, in Fig. 10(b) and (c), it can be seen that the end-to-end tree delay of the proposed ODQMRP-PSPT is superior to that of the SPT strategy with the growth of the size of the multicast group, which means that the proposed ODQMRP-PSPT is more suitable for the applications with the greater demand on delay, for example, the video teleconferencing. 


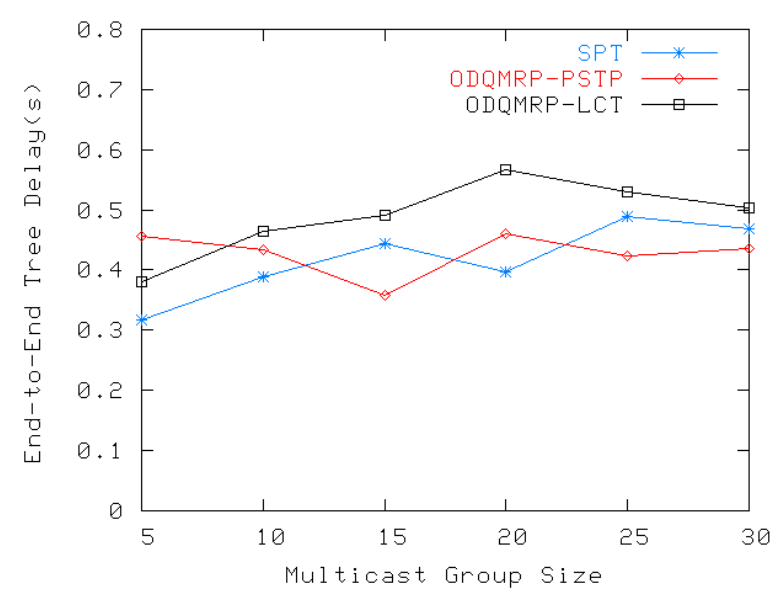

(a) QoS Class 0 .

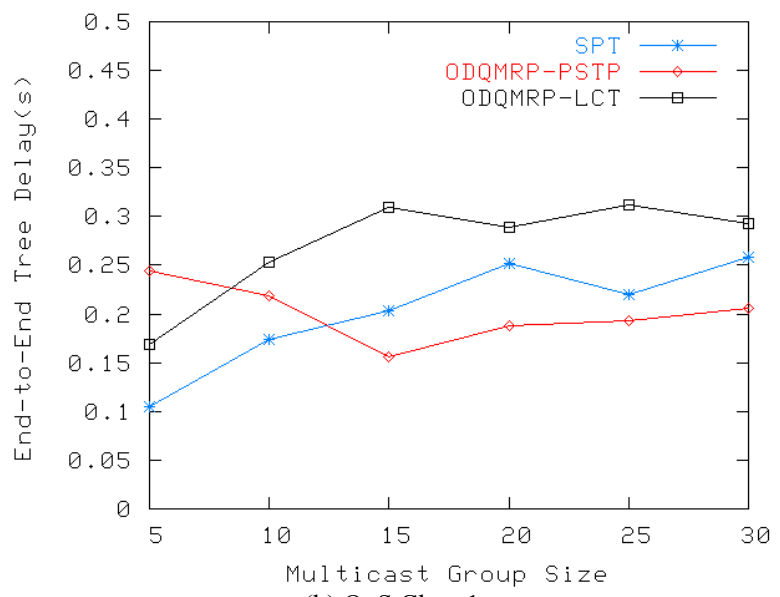

(b) QoS Class 1 .

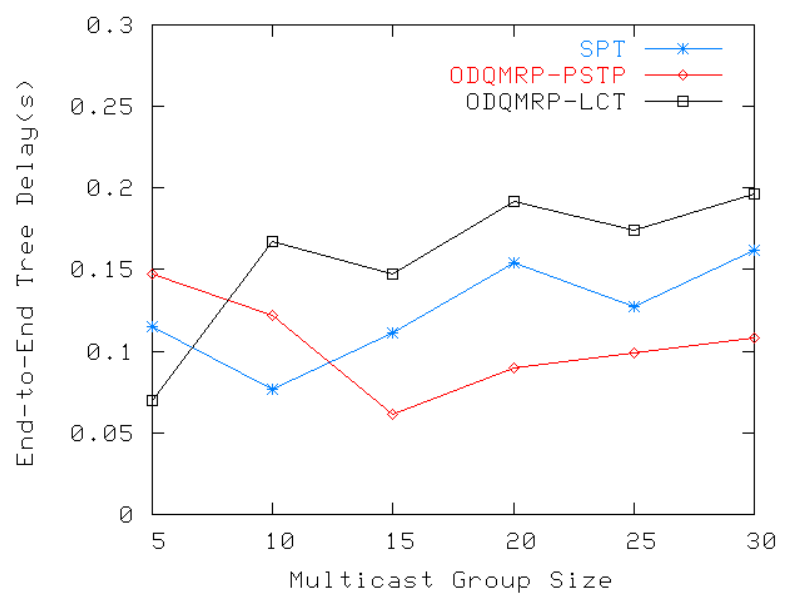

(c) QoS Class 2 .

Figure 10. Performance comparison of the end-to-end tree delay of the SPT strategy and the proposed ODQMRP under different QoS Classes.

Figure 11 demonstrates the comparison of the tree cost versus the multicast group size between the SPT strategy and the proposed ODQMRP for different QoS Classes. In Fig. 11(a), (b), and (c), in terms of overall performance, the tree cost of the proposed ODQMRP-LCT is better than that of the ODQMRP-PSPT and the SPT strategy. This can be explained by the fact that the proposed ODQMRP-LCT focuses on the optimization of the tree cost in the construction of the multicast tree under the condition that the QoS constraints, i.e., the delay requirement and the available bandwidth requirement, are guaranteed. However, the SPT strategy or the proposed ODQMRP-PSPT only optimizes the delay constraint, although the proposed ODQMRP-PSPT takes the available bandwidth requirement into account.

Moreover, the tree cost of the proposed ODQMRPPSPT is superior to that of the SPT strategy with the range of 15 to 30 of the multicast group size, which indicates that the performance of the proposed ODQMRP-PSPT is better than that of the SPT strategy as the increase of the size of the multicast group.

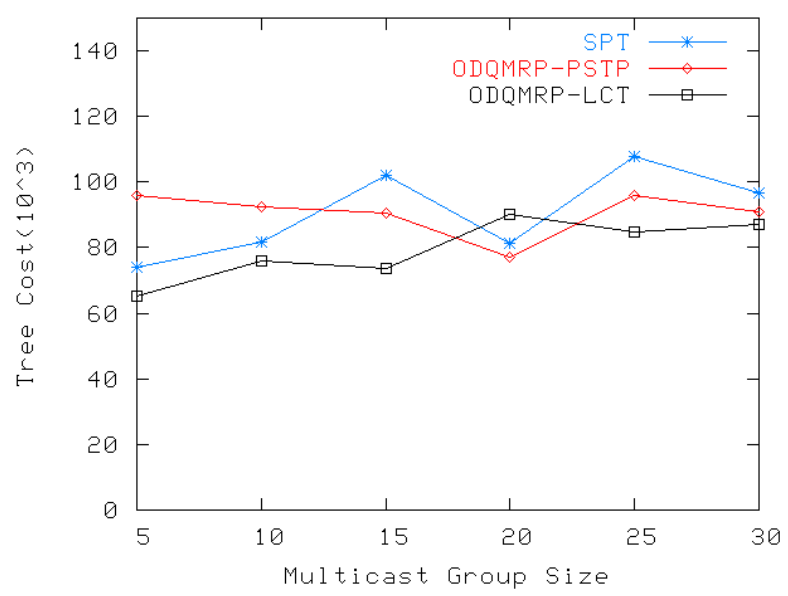

(a) QoS Class 0.

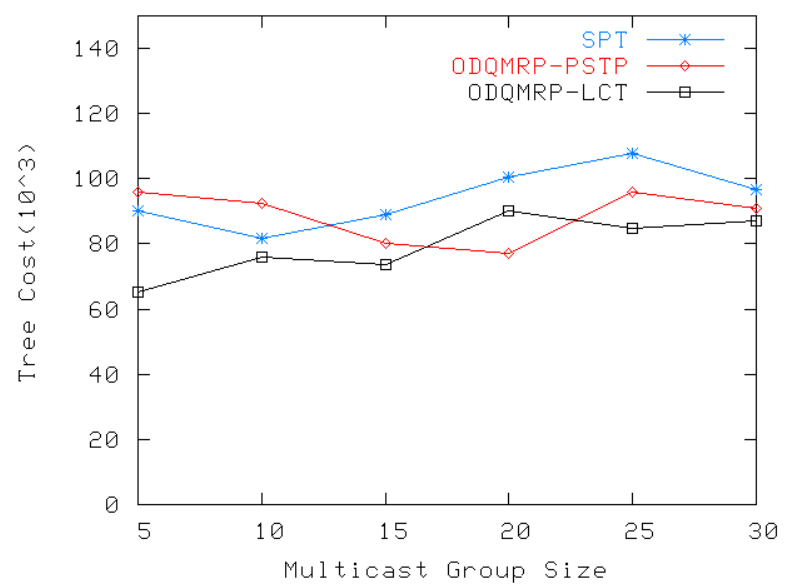

(b) QoS Class 1.

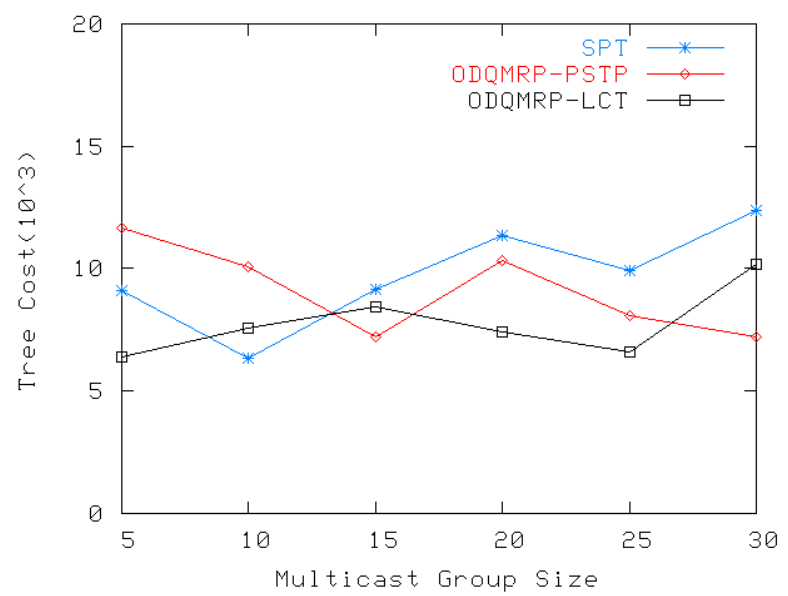

(c) QoS Class 2.

Figure 11. Performance comparison of the tree cost of the SPT strategy and the proposed ODQMRP under different QoS Classes. 
Figure 12 compares the failure ratio of multicasting connections versus the multicast group size between the SPT strategy and the proposed ODQMRP for different QoS Classes. In Fig. 12(a), (b), and (c), we can observe that the performance of the failure of multicasting connections of our proposed ODQMRP-LCT and ODQMRP-PSPT surpasses that of the SPT strategy in terms of the overall performance, which demonstrates that as the multicast group size increases, the success ratio of the QoS multicasting requests of the proposed ODQMRP is superior to that of the SPT strategy. For that reason, the proposed ODQMRP can easily establish the QoS multicasting connections. This can be explained by the fact that the proposed the SPT strategy does not take into account the available bandwidth constraint, which results in the higher possibility in the failure of QoS multicasting connections.

Furthermore, from Fig. 12(a) and (c), it can be easily seen that the failure ratio of multicasting connections of the proposed ODQMRP-LCT is much lower than that of the SPT strategy and the ODQMRP-PSPT in the range of 15 to 30 of the multicast group size. This illustrates that the proposed ODQMRP-LCT is more appropriate to the applications with the less available bandwidth, for example, the video teleconferencing and high-speed data on-demand.

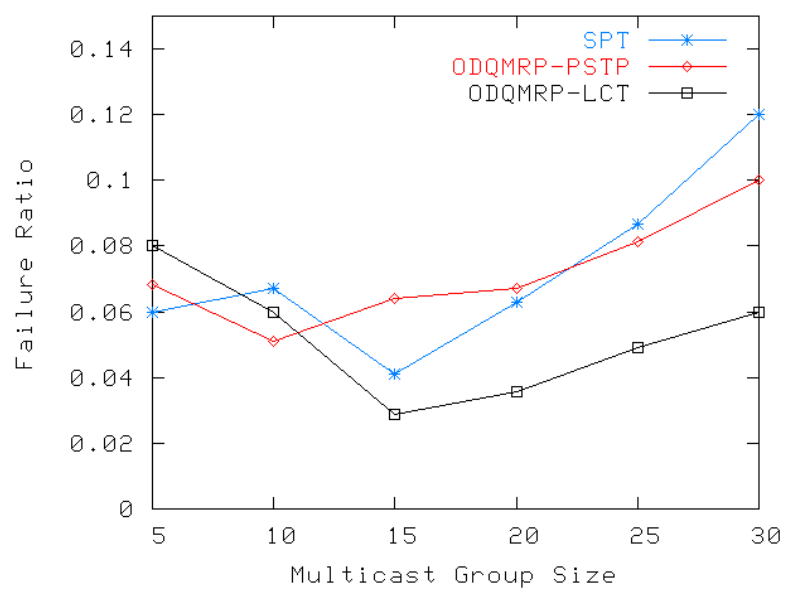

(a) QoS Class 0

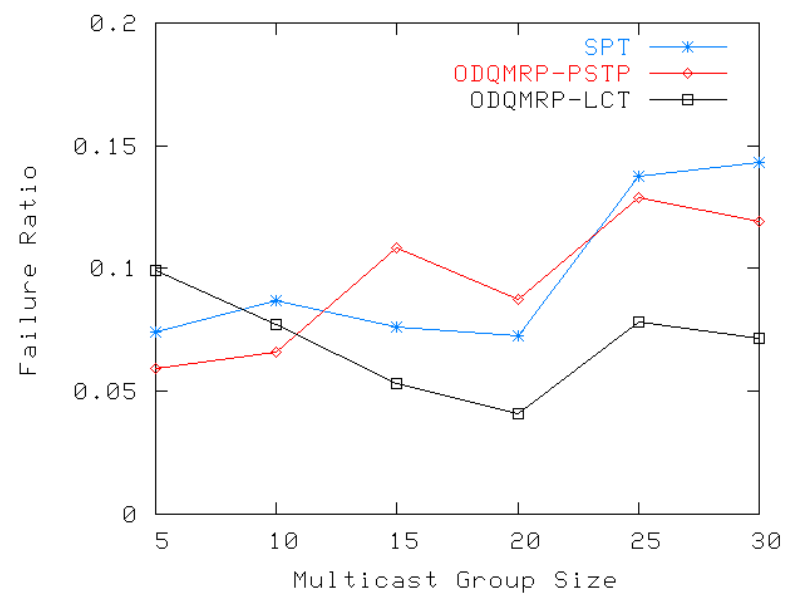

(b) QoS Class 1 .

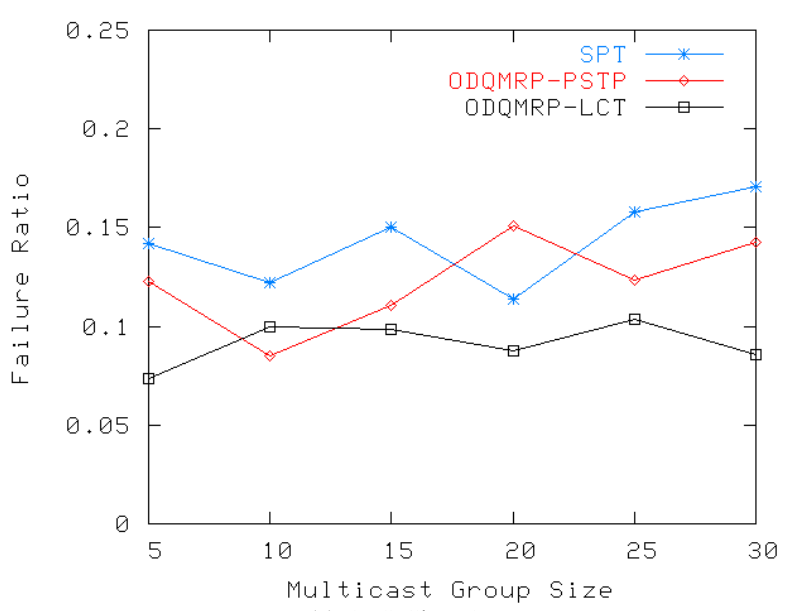

(c) QoS Class 2

Figure 12. Performance comparison of the failure ratio of the SPT strategy and the proposed ODQMRP under different QoS Classes.

\section{CONCLUSIONS}

In this paper, aiming at the difficulty to provide the coverage over the special regions or the areas of high latitudes by the existing hierarchical satellite networks, we introduce a novel triple-layered LEO/HEO/GEO satellite network architecture including three satellite layers, i.e., the LEO layer, the HEO layer, and the GEO layer, which provides the near-global coverage with 24 hour uninterrupted over the areas varying from $75^{\circ} \mathrm{S}$ to $90^{\circ} \mathrm{N}$. On the basis of this novel architecture, we propose an on-demand QoS multicast routing protocol (ODQMRP) for satellite IP networks by employing the concept of logical locations to isolate the mobility of LEO and HEO satellites. In the proposed ODQMRP, we present two strategies, i.e., the PSPT strategy and the LCT strategy, to create the multicast trees under the condition that the QoS constraints, containing the delay requirement, and the available bandwidth requirement, are both guaranteed. Moreover, the main goal of the PSPT strategy and the LCT strategy is to minimize the path delay and the path cost of the multicast trees, respectively. Simulation results demonstrate that the performance benefits of ODQMRP in terms of three performance metrics, i.e., the end-to-end tree delay, the tree cost, and the failure ratio of multicasting connections in contrast with the traditional non-QoS guaranteed shortest path tree (SPT) strategy.

\section{ACKNOWLEDGMENT}

The authors would like to acknowledge the support from the National Natural Science Foundation of China under Grant No. 61003250 and No. 60902042, the National Research Foundation for the Doctoral Program of Higher Education of China under Grant No. 20090006110014, and the Beijing Municipal Natural Science Foundation under Grant No. 4102042.

\section{REFERENCES}


[1] B. R. Elbert, The Satellite Communication Applications Handbook, 2nd ed., Norwood, MA: Artech House, 2004, pp. 14-26.

[2] T. H. Nguyen and M. N. O. Sadiku, "Next generation networks", IEEE Potentials, vol. 21, no. 2, pp. 6-8, Apr. /May. 2002.

[3] International Telecommunication Union, "Terms of reference of ITU-T focus group on future networks", [Online]. http://www.itu.int/oth/T3A02000001/en

[4] P. Chitre and F. Yegenoglu, "Next-generation satellite networks: architectures and implementations", IEEE Communications Magazine, vol. 37, no. 3, pp. 30-36, Mar. 1999.

[5] I. F. Akyildiz and S. Jeong, "Satellite ATM networks: a survey", IEEE Communications Magazine, vol. 35, no. 7, pp. 30-43, Jul. 1997.

[6] G. Akkor, "Multicast communication support over satellite networks", Ph.D. dissertation, Department of Electrical and Computer Engineering, University of Maryland, College Park, MD, 2005.

[7] L. Wood, A. Clerget, I. Andrikopoulos, G. Pavlou, and W. Dabbous, "IP routing issues in satellite constellation networks", International Journal of Satellite Communications, vol. 19, no. 1, pp. 69-92, Jan. /Feb. 2001

[8] M. Werner, C. Delucchi, H. Vogel, G. Maral, and J. De Ridder, "ATM-based routing in LEO/MEO satellite networks with intersatellite links", IEEE Journal on Selected Areas in Communications, vol. 15, no. 1, pp. 69 82, Jan. 1997.

[9] M. Werner, G. Berndl, B. Edmaier, "Performance of optimized routing in LEO intersatellite link networks", in Proc. IEEE VTC'97, vol. 1, May. 1997, 246-250.

[10] H. S. Chang, B. W. Kim, C. G. Lee, S. L. Min, Y. Choi, H. S. Yang, D. N. Kim, and C. S. Kim, "FSA-based link assignment and routing in low-Earth orbit satellite networks", IEEE Transactions on Vehicular Technology, vol. 47, no. 3, pp. 1037-1048, Aug. 1998.

[11] H. Uzunalioglu, I. F. Akyildiz, Y. Yesha, and W. Yen, "Footprint handover rerouting protocol for low Earth orbit satellite networks", Wireless Networks, vol. 5, no. 5, pp. 327-337, Sept. 1999.

[12] H. Uzunalioglu, "Probabilistic routing protocol for low Earth orbit satellite networks", in Proc. IEEE ICC'98, Jun. 1998, pp. 89-93.

[13] K. Tsai and R. P. Ma, "DARTING: a cost-effective routing alternative for large space-based dynamic-topology networks", in Proc. IEEE MILCOM'95, vol. 2, Nov. 1995, pp. 682-686.

[14] R. A. Raines, R. F. Janoso, D. M. Gallagher, and D. L. Coulliette, "Simulation of two routing protocols operating in a low Earth orbit satellite network environment", in Proc. IEEE MILCOM'97, vol. 1, Nov. 1997, pp. 429-433.

[15] T. R. Henderson and R. H. Katz, "On distributed, geographic-based packet routing for LEO satellite networks", in Proc. IEEE GLOBECOM'00, vol. 2, Nov. /Dec. 2000, pp. 1119-1123.

[16] E. Ekici, I. F. Akyildiz, and M. D. Bender, "A distributed routing algorithm for datagram traffic in LEO satellite networks", IEEE/ACM Transactions on Networking, vol. 9, no. 2, pp. 137-147, Apr. 2001.

[17] I. F. Akyildiz, E. Ekici, and M. D. Bender, "MLSR: a novel routing algorithm for multilayered satellite IP networks", IEEE/ACM Transactions on Networking, vol. 10, no. 3, pp. 411-424, Jun. 2002.
[18] C. Chen and E. Ekici, "A routing protocol for hierarchical LEO/MEO satellite IP networks", Wireless Networks, vol. 11, no. 4, pp. 507-521, Jul. 2005.

[19] B. Quinn and K. Almeroth, "IP multicast applications: challenges and solutions", Internet RFC 3170, Sept. 2001.

[20] L. H. Sahasrabuddhe and B. Mukherjee, "Multicast routing algorithms and protocols: a tutorial", IEEE Network, vol. 14, no. 1, pp. 90-102, Jan. /Feb. 2000.

[21] P. Paul and S. V. Raghavan, "Survey of multicast routing algorithms and protocols", in Proc. 15th International Conference on Computer Communication, vol. 1, Aug. 2002, pp. 902-926.

[22] U. Varshney, "Multicast over wireless networks", Communications of the ACM, vol. 45, no. 12, pp. 31-37, Dec. 2002.

[23] D. Waitzman, C. Partridge, and S. E. Deering, "Distance vector multicast routing protocol”, Internet RFC 1075, Nov. 1988.

[24] A. Ballardie, "Core based trees (CBT version 2) multicast routing", Internet RFC 2189, Sept. 1997.

[25] S. Deering, "Host extensions for IP multicasting", Internet RFC 1112, Aug. 1989.

[26] J. Moy, "Multicast routing extensions for OSPF", Communications of the ACM, vol. 37, no. 8, pp. 61-66, Aug. 1994.

[27] E. M. Royer and C. E. Perkins. "Multicast operation of the ad hoc on-demand distance vector routing protocol", in Proc. ACM MOBICOM,'99 Aug. 1999, pp. 207-218.

[28] C. K. Toh, G. Guichal, and S. Bunchua, "ABAM: ondemand associativity-based multicast routing for ad hoc mobile networks", in Proc. IEEE VTC'00, vol. 3, Sept. 2000, pp. 987-993.

[29] S. Lee, W. Su, and M. Gerla, "On-demand multicast routing protocol (ODMRP) for ad hoc networks", Internet Draft, Jul. 2000.

[30] J. J. Garcia-Luna-Aceves and E. L. Madruga, "The coreassisted mesh protocol", IEEE Journal on Selected Areas in Communications, vol. 17, no. 8, pp. 1380-1394, Aug. 1999.

[31] E. Ekici, I. F. Akyildiz, and M. D. Bender, "A multicast routing algorithm for LEO satellite IP networks", IEEE/ACM Transactions on Networking, vol. 10, no. 2, pp. 183-192, Apr. 2002.

[32] D. Yang and W. Liao, "On multicast routing using rectilinear Steiner trees for LEO satellite networks", IEEE Transactions on Vehicular Technology, vol. 57, no. 4, pp. 2560-2569, Jul. 2008.

[33] L. Chen, J. Zhang, and K. Liu, "Core-based shared tree multicast routing algorithms for LEO satellite IP networks", Chinese Journal of Aeronautics, vol. 20, no. 4, pp. 353361, Aug. 2007.

[34] I. F. Akyildiz, E. Ekici, and G. Yue, "A distributed multicast routing scheme for multi-layered satellite IP networks", Wireless Networks, vol. 9, no. 5, pp. 535-544, Sept. 2003.

[35] S. Kota and M. Marchese, "Quality of service for satellite IP networks: a survey", International Journal of Satellite Communications and Networking, vol. 21, no. 4-5, pp. 303-349, Jul. 2003.

[36] Y. Zhou, F. Sun, and B. Zhang, "A novel QoS routing protocol for LEO and MEO satellite networks", International Journal of Satellite Communications and Networking, vol. 25, no. 6, pp. 603-617, Sept. 2007.

[37] H, Xu, F. Huang, and S. Wu, "A distributed QoS routing based on ant algorithm for LEO satellite network", Journal of Electronics, vol. 24, no. 6, pp. 765-771, Nov. 2007. 
[38] P. Wang, X. Gu, and G. Liu, "Multi-QoS routing for LEO satellite networks", in Proc. 9th International Conference on Advanced Communication Technology, vol. 1, Feb. 2007, pp. 728-731.

[39] O. Ercetin, S. Krishnamurthy, S. Dao, and L. Tassiulas, "A predictive QoS routing scheme for broadband low Earth orbit satellite networks", in Proc. 11th IEEE International Symposium on Personal, Indoor and Mobile Radio Communications, vol. 2, Sept. 2000, pp. 1064-1074.

[40] K. Kimura, K. Inagaki, and Y. Karasawa, "Double-layered inclined orbit constellation for advanced satellite communications network", IEICE Transactions on Communications, vol. E80-B, no. 1, pp. 93-102, Jan. 1997.

[41] L. Chin, J. Chang, and C. Huang, "Performance of a twolayer LEO satellite communication network", IEEE Transactions on Aerospace and Electronic Systems, vol. 33, no. 1, pp. 225-231, Jan. 1997.

[42] L. Wood, "Internetworking with satellite constellations", $\mathrm{Ph} . \mathrm{D}$. dissertation, School of Electronics, Computing and Mathematics, University of Surrey, Guildford, United Kingdom, 2001.

[43] C. Perkins, E. Belding-Royer, and S. Das, "Ad hoc ondemand distance vector (AODV) routing", Internet RFC 3561, Jul. 2003.

[44] J. A. Bondy and U. S. R. Murty, Graph Theory with Applications, Great Britain: The Macmillan Press, 1976, pp. 15-20.

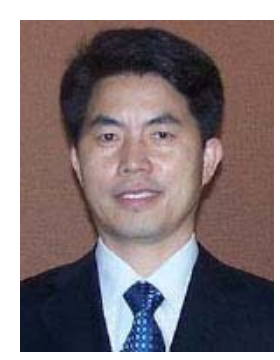

Zhizhong Yin received his B.S. degree from the Academy of Equipment Command \& Technology, Beijing, China in 1988, and his M.S. degree in digital multimedia engineering from the School of Information Engineering, University of Science and Technology Beijing, Beijing, China in 2002. He is currently working toward the Ph.D degree in communication and information systems at the Department of Communication Engineering, School of Information Engineering, University of Science and Technology Beijing, Beijing, China. He has also been a senior engineer at the Academy of Equipment Command \& Technology, Beijing, China. His current research interests include satellite networks, broadband wireless communications, cognitive radio networks, mobile computing, and next generation networks.

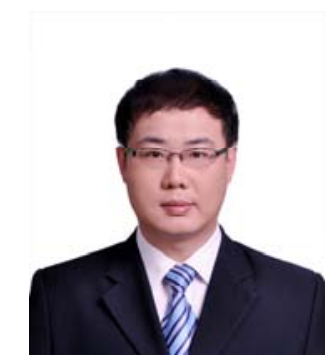

Long Zhang received his B.S. degree in communication engineering from the Faculty of Information Engineering, China University of Geosciences, Wuhan, China in June 2006. He is currently working toward the Ph.D degree in communication and information systems at the Department of Communication Engineering, School of Information Engineering, University of Science and Technology Beijing, Beijing, China. His current research interests include deep space information networks, delay and disruption tolerant networks, cognitive radio, satellite and space communications, mobile ad hoc networks, and future information networks.

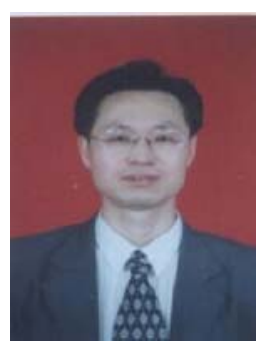

Xianwei Zhou received his B.S. degree in Department of Mathematics from the Southwest China Normal University, Chongqing, China in 1986, and his M.S. degree in Department of System Science and Mathematics from Zhengzhou University, Zhengzhou, China in 1992, and in 1999, he obtained the Ph.D. degree in Department of Transportation Engineering from the Southwest Jiaotong University, Chengdu, China. He was engaged in postdoctor study in information and communication engineering at the School of Electronic and Information Engineering, Beijing Jiaotong University, Beijing China, from 1999 to 2000. In 2001, he joined the Department of Communication Engineering, School of Information Engineering, University of Science and Technology Beijing, Beijing, China, where he is currently a professor and Ph.D. supervisor. So far he has published over 100 research papers in the domestic and international scientific journals and conferences. His current research interests include security of communication networks, next generation networks, cognitive radio, mobile IPv6, scheduling theory, and game theory. 